\title{
Efficient Non-Invasive Registration with A-mode Ultrasound in Skull Surgery
}

\author{
Aleksandra Popovic ${ }^{1}$, Stefan Heger ${ }^{1}$, Axel Follmann ${ }^{1}$, Ting $\mathrm{Wu}^{1}$, \\ Martin Engelhardt ${ }^{2}$, Kirsten Schmieder ${ }^{2}$ and Klaus Radermacher ${ }^{1}$ \\ RWTH Aachen University, Ruhr-University Bochum \\ Germany
}

\section{Introduction to Neuronavigation}

Surgical navigation is an interactive localization technique to establish a relation between surgical instruments, patient's anatomy, and additional data (e.g. preoperative or intraoperative patient images or atlases).

The first attempt to localize structures within the brain using orientation points on the skull surface and a standardized brain atlas was done at the beginning of 20th century (1908) by Sir Victor Horsley and Robert H. Clarke (Tan \& Black 2002). They used a rigid frame (Horsley-Clarke apparatus) designed to measure salient points on the skull in Cartesian 3D coordinate system and transform them to the coordinate system of a brain atlas. Coordinate system computations allowed a surgeon to position a tool to a desired position within the skull. Findings of Horsley and Clarke were followed by further improvements in frame design, coordinates computations, and brain atlases. However, the major breakthrough was achieved as imaging technologies emerged, Computed Tomography (CT) in 1973 and Magnetic Resonance Imaging (MRI) during 1980s. Three-dimensional brain images allowed an extension of stereotactic computation to the entire intracranial space. Until the end of 1990s, stereotaxy has been the most common localization technique in the neurosurgery (Grunert et al. 2003). Frameless navigation emerged as an alternative to conventional stereotactic surgery, in order to decrease invasiveness and to improve localization and targeting. The main objective of image-guided surgery is to track surgical instruments in relation to the patient images. To achieve this, a geometric transformation between physical and image space has to be established in a registration procedure. First attempts to avoid invasive stereotactic frames in neurosurgical navigation emerged at the end 1980s with neuronavigator arms (Watanabe et al. 1987, Watanabe 1996, Laborde et al. 2002), sixdimensional mechanical digitizers performing point-based registration of the patient and image space and computer-based arm tracking. The main disadvantage of those systems is the need for repeated registration after each repositioning of the patient's head (Schiffbauer 1992). Further development of digitalization system introduced magnetic (Tan et al. 1993) and optical localizers (Zamorano et al. 1992;1993) in the neuronavigation, able to interactively track instruments and patient position. Although optical tracking devices have a disadvantage of requiring permanent line-of-sight during tracking, they are currently state-of-the-art technique in surgical navigation due to a better localization accuracy 
compared to the magnetic systems. Commercially available optical neuronavigation systems include StealthStation $\odot$ (Medtronic, USA) and Vector Vision@cranial (BrainLab, Germany). The technique in which an operator freely moves tracked surgical instruments is referred to as a free-hand navigation. Although image-guidance combined with a preoperative planning allows a better spatial orientation through the position feedback, the positioning accuracy is restrained by human factors, such as hand-eye coordination. For some deep brain targeting applications requiring high precision, frame-based stereotaxy is still preferred to free-hand image-guidance (Spivak and Pirouzmand 2005). An alternative approach is to use a robotic system, providing a high spatial accuracy, an ability to perform time-lasting repetitive movements, and high movement predictability (Nathoo 2005). In recent years, research efforts have been made to introduce robotic systems in the neurosurgical practice (Bai et al. 2001, Federspil et al. 2003, Federspil and Plinkert 2004, Handini et al. 2004, Bast et al. 2006).

\section{Motivation - CRANIO System for Robot and Computer Aided Neurosurgery}

Osseous tumors of the calvaria are rare diseases presented with various histological and imaging findings (Arana 2004, Engelhardt 2006). The majority of calvarial tumors encountered in the field of neurosurgery are either meningiomas or metastases. They are usually treated by a total or subtotal recision followed by a removal of the tumorous tissue.

The CRANIO system for computer and robot assisted craniectomy (Bast et al. 2006) is being developed for the surgical treatment of patients suffering from calvarial tumors, accompanied by preoperative design and manufacturing of individual implants for immediate cranial reconstruction, also called cranioplasty ( $\mathrm{Wu}$ et al. 2006). Clinical motivation for the robotic craniectomy (removal of a cranial tumor) is twofold:

- A robotic removal of the cancerous bone is significantly more time-efficient than manual procedures. Laboratory experiments showed 50\%-70\% milling time reduction in comparison to the manual micro-milling (Bast et al. 2003). This might lead to a decrease in operating time with benefits to patient's health and cost reduction. Furthermore, the operating surgeon is relieved from a tenacious instrument holding.

- In order to preoperatively manufacture an individual implant and perform cranioplasty immediately following a tumor ablation, the resection geometry has to be known prior to the operation and has to be accurately reproduced during the operation. Free-hand neurosurgical milling, even with a navigational help, cannot meet the accuracy requirements.

To achieve these objectives, all aspects of the computer aided surgery have to be addressed: segmentation (Popovic et al. 2006), resection planning, milling path generation and simulation (Popovic et al. 2003), implant planning and manufacturing (Wu et al. 2006), intraoperative navigation and robot control (Popovic et al. 2003, See Figure 1). Intraoperatively, a geometrical relation between patient's physical space and preoperative data (e.g. the resection path in coordinate system of the CT scanner) has to be established in the process of an intraoperative registration (see section 2.3.1). Accuracy of execution of the preoperative plan intraoperatively depends on three factors:

1. Patient registration accuracy, i.e. transformation accuracy of the planed milling path from the coordinate system of the model data to the in situ coordinate system of the patient. 
2. Robot registration accuracy, i.e. transformation accuracy of the planed milling path from the coordinate system of patient to the robot coordinate system.

3. Robot accuracy, i.e. the positioning accuracy of the robot.

Due to the mechanical precision and reproducibility of the robotic system, the patient registration is the most accuracy-critical part of the system. Apart from accuracy requirements, time efficiency, reliability and intuitiveness of the registration process are crucial for application of the system in clinical routine. The problem of simultaneous accuracy improvement and invasiveness reduction can be solved using A-Mode Ultrasound (AUS) based registration.

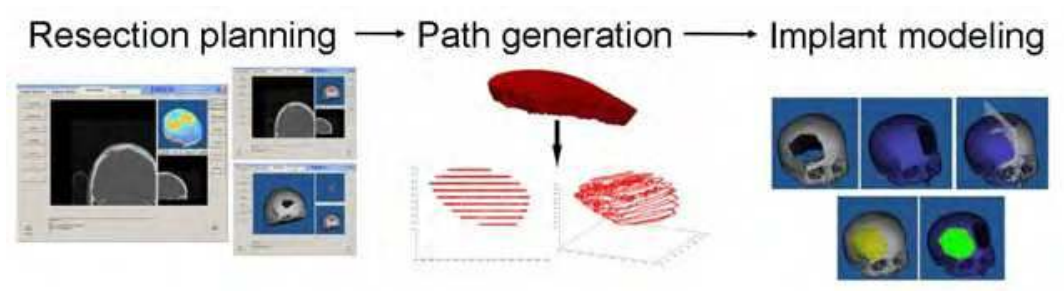

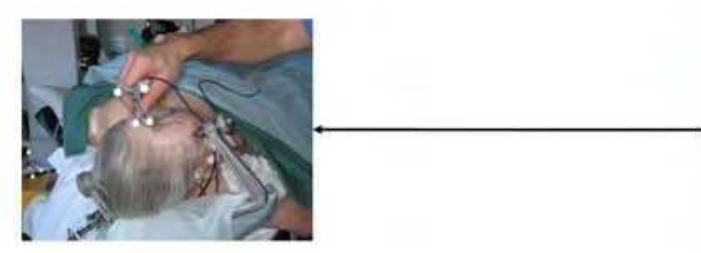

Navigation

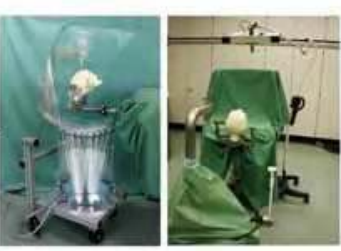

Robotic surgery

Figure 1. The concept of the CRANIO system

\section{Registration algorithms}

The process of patient registration involves finding a transformation relating the patient's physical space with the coordinate system(s) of preoperative and/or intraoperative acquired images. The registration procedure is an optimization method searching for an optimal transformation between the points in patient's physical space, i.e. measurement points $\left\{\vec{m}_{i}\right\}$ and points in image space, i.e. data points $\left\{\vec{d}_{i}\right\}$. Therefore, the transformation is represented as:

$$
T: \vec{m} \mapsto \vec{d} \Leftrightarrow T(\vec{m})=\vec{d},
$$

where $\vec{m}$ is a measurement vector with $\vec{m}=\left[\vec{m}_{0} \ldots \vec{m}_{N-1}\right]^{T}, \vec{d}=\left[\vec{d}_{0} \ldots \vec{d}_{N-1}\right]^{T}$ a data vector and $\mathrm{N}$ the number of points. If $\mathrm{T}$ is a linear, rigid transformation, it represents a rotation and a translation of the points:

$$
T\left(\vec{m}_{i}\right)=R \cdot \vec{m}_{i}+\vec{t},
$$

where $\mathrm{R}$ is a $3 \times 3$ rotation matrix and $\mathrm{t}$ is a $3 \times 1$ translation vector. 


\subsection{Point based registration}

Point based registration refers to problems of finding a transformation between two coordinate systems using pairs of points in both systems, i.e. if the number of measurement and data points is the same and there exists a unique correspondence between the points. Those points are referred to as homologous markers to point out pair wise point correspondence. In a general case, the transformation may include a scaling. However, since the goal of registration for neurosurgical navigation is a registration between the images and the patient's space for the same patient, a linear, rigid transformation is assumed.

Point based registration is an instance of the orthogonal Procrustes optimization problem. As mentioned above, the objective is to minimize the transformation error. A first step is to replace the points with their demeaned values:

$$
\begin{aligned}
\vec{m}^{m} & =\frac{1}{N} \sum_{i=0}^{N-1} \vec{m}_{i} \\
\vec{d}^{m} & =\frac{1}{N} \sum_{i=0}^{N-1} \vec{d}_{i} \\
\vec{m}_{i}^{m} & =\vec{m}_{i}-\vec{m}^{m} \\
\vec{d}_{i}^{m} & =\vec{d}_{i}-\vec{d}^{m}
\end{aligned}
$$

The demeaned measurement and data points can be arranged in two NxD matrices, $\mathbf{M}$ and $\mathrm{D}$ respectively, $\mathrm{N}$ being the number of points and $\mathrm{D}$ being the dimension of data. The $\mathrm{DxD}$ correlation matrix:

$$
K=M^{T} D
$$

represents the goodness of prediction of the data points from the measurement points. Using the Singular Value Decomposition (SVD), the matrix K can be represented as:

$$
K=U \cdot \Sigma \cdot V^{T}
$$

Where $\mathrm{U}$ and $\mathrm{V}$ are DxD orthogonal matrices and $\Sigma$ is a DxD diagonal matrix.

Finally, the rotation matrix representing a rotation of measurement points in the coordinate system of the data points is:

$$
R=V \cdot \Delta \cdot U^{T}
$$

where

$$
\Delta=\operatorname{diag}\left(1,1, \operatorname{det}\left(V U^{T}\right)\right)
$$

The translation is computed as a vector of distance between centres of mass of measurement and data points:

$$
t=\vec{d}^{m}-R \vec{m}^{m}
$$

If the number of points is three ( $N=3)$, the SVD is uniquely defined. For $N>3$, the system is over-defined and the singular value decomposition is not a trivial task. 


\subsection{Surface based registration}

Surface based registration uses a surface representation of at least one of the initial sets (measurement or data points). In the neurosurgical registration with CT images, a natural way to represent data points is a bone surface extracted from the 3D-CT images of the patient. Intraoperativelly, using the A-mode US probe, a significantly fewer number of bone surface points can be obtained. Therefore, the surface registration problem for this application is de facto a surface to points matching. The iterative closest point (ICP) is a widely used algorithm for this kind of matching issues. The ICP was proposed by Besl and McKey for the registration of 3D shapes (Besl and McKey 1992). The algorithm works with different surface representations. For this application, a point set surface representation is assumed.

The ICP is an iterative procedure with two phases: detection of closest point on the surface and least squares registration. If $D$ is a bone surface from $C T$ images, the closest point from a measurement point $\mathrm{m}_{\mathrm{i}}$ is:

$$
d_{i}\left(\vec{m}_{i}, D\right)=\min _{\vec{x} \in D}\left(\left\|\vec{x}-T_{0}\left(\vec{m}_{i}\right)\right\|\right)
$$

where $T_{0}$ is an initial transformation. As the result, $N$ pairs of associated points $\left\{\mathrm{m}_{\mathrm{i}}, \mathrm{d}_{\mathrm{i}}\right\}$ are obtained, where $\mathrm{N}$ is the number of measurement points. Afterwards, a point based least squares registration is performed, as described in section 3.1. The iterations are repeated until one of the following conditions is met: a convergence is reached or the number of iterations exceeded the predefined maximal iterations number. An obvious advantage of the ICP algorithm is that a correspondence between the measurement and the data points is computed. This is a particularly important issue influencing total registration time in the operating theatre. Therefore, if the ICP algorithm is used, the number of points $(\mathrm{N})$ can be significantly higher than in the case of point based matching.

\subsection{Registration errors}

The goal of the registration is to find an optimal transformation $T$, such that the transformation error between the two coordinate systems is minimal. In an ideal case, a transformation maps the measurement points exactly to the data points. Otherwise, the registration error is:

$$
F R E=\sqrt{\frac{1}{N} \sum_{i=0}^{N-1}\left(T\left(\vec{m}_{i}\right)-\vec{d}_{i}\right)^{2}}
$$

This error is usually referred to as the Fiducial Registration Error (FRE). For the most clinical applications, a more relevant error is the Target Registration Error (TRE) showing the error in remote surgical target points. The TRE depends on FRE and on the position of a target in relation to the data points. According to the statistical analysis from (Fitzpatrick 1998), TRE is proportional to the distance between the target point and the principal axis of the points and reverse-proportional to distance between the fiducial points and their axes. From these considerations, heuristics considering selection of the points can be drawn. The configuration of fiducials should follow a regular pattern with isometric axes, e.g. a tetrahedron, in order to avoid a large deviance between the target and the principal axes. This conclusion can be applied to both Procrustes point based registration and ICP surface based registration. In further text, as the registration error, TRE will be assumed. 


\section{Registration techniques}

The essential factor influencing accuracy of a neuronavigational system is the type and the position of the used markers. Implantable bone screws (BS) offer high precision, since their identification in CT-images and operating site is trivial. However, this approach implies significant additional efforts for the clinical team concerning the clinical work flow (including an additional operation and anaesthesia) as well as discomfort for the patients, skull or skin infection including postoperative pain. Adhesive skin markers (SM) are a more convenient alternative and are widely used (overview in Steinmeier et al. 2000 and Bernardete et al. 2001), although they induce a decreased accuracy through the skin shift, especially due to the immobilization of the patient with a clamp (Wolfberger et al. 2002). Furthermore, the placement of both types of markers requires trained personnel and partial shaving of the patient. (Wolfsberger et al. 2002) proposed a set of anatomical landmarks (AL) for point to point registration, and reported an error of $3.2 \pm 1.1 \mathrm{~mm}$ using five anatomical landmarks.

In contrast, surface based methods may be inaccurate as far as registration is restricted to the very limited bone surface area of the surgical approach. Using a transcutaneous A-mode ultrasound (US) registration of anatomic landmarks and surface areas underneath the soft tissue may be a solution overcoming displacement problems without increasing the invasiveness (Mauerer 1999 et al., Heger et al. 2005). Furthermore, the ultrasonic registration avoids the need for the preoperative segmentation of the markers. The approach to achieve a time efficient and robust registration by a combination of an optimized man-machineinteraction strategy, signal processing and different registration techniques has been reported in detail in (Heger et al. 2005). Its application in skull surgery is presented here. A clinical protocol is developed for US-based registration, namely concerning the selection of adequate numbers and positions of points to be palpated.

\begin{tabular}{l|cccc}
\hline $\begin{array}{l}\text { Technique/ } \\
\text { Feature }\end{array}$ & $\begin{array}{c}\text { Bone Screws } \\
\text { (BS) }\end{array}$ & $\begin{array}{c}\text { Adhesive } \\
\text { Skin Markers } \\
(\mathrm{SM})\end{array}$ & $\begin{array}{c}\text { Anatomical } \\
\text { Landmarks } \\
(\mathrm{AL})\end{array}$ & $\begin{array}{c}\text { Surface } \\
\text { points }\end{array}$ \\
\hline Accuracy & ++ & - & - & + \\
Invasiveness & --- & + & + & + \\
Patient discomfort & -- & +- & + & + \\
Palpation area & + & + & + & - \\
Additional & - & - & +- & + \\
Training of the OP staff & - & & & \\
\hline
\end{tabular}

Table 1. Cross-comparison of the clinical and technical features

\section{Registration approaches}

Three registration approaches have been investigated: point-to-point matching, direct surface-based matching and US-based surface matching. The tests are performed in the following modes:

- $\quad$ Mode I

(a) Point based registration with implanted markers

(b) Point based registration with skin adhesive markers 
- $\quad$ Mode II

(a) Point based registration with anatomical landmarks, followed by

(b) Surface-based registration, directly on the bone surface

- $\quad$ Mode III

Point based registration with anatomic landmarks, followed by transcutaneous surfacebased matching, using the US probe

Surface-based registration (IIb) is done by palpating points in skull convexity and facial structures and fitting them to the 3D model obtained from CT imaging study of the patient. Surface based registration is done using a pre-registration with anatomical landmarks followed by the ICP algorithm. Combining the ICP algorithm with different registration techniques (SM or BS) would not address the issue of invasiveness reduction and is therefore omitted.

For AL registration, using both previous experience and guidelines from the literature (Wolfsberger et al. 2002), following anatomical landmarks are used: the most posterior point in the root of the nose, the most anterior point at the nose tip, and the most anterior part of frontozygomatic suture (left and right). Surface palpated points were defined as follows: three lateral left, three lateral right, four on the frontal skull convexity, four on the forehead, and four periorbital, summing up to total 18 surface points for the ICP algorithm.

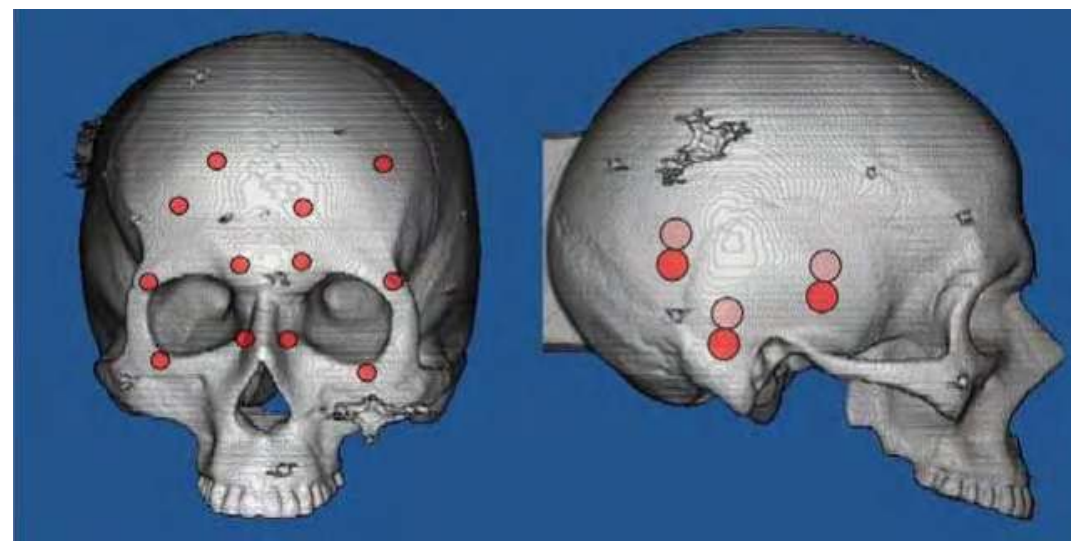

Figure 3. Surface registration protocol with 18 points

The palpation protocol is shown in Figure 3. A limiting factor for the selection of palpation surfaces is patient fixation in a Mayfield-clamp. A pre-evaluation of standard fixation techniques and postures has been performed prior to the registration trials, in order to detect unapproachable structures. This analysis resulted in a palpation protocol excluding posterior and caudal cranium. Furthermore, although highly distinctive, the jaws were omitted, since the jaws are normally not scanned in a standard radiological protocol for neurosurgery.

\section{Experimental setup}

The registration approaches described in section 4 have been evaluated in three settings:

- $\quad$ Phantom laboratory trials with a Sawbone® solid foam model.

- Anatomical laboratory trials with a formalin fixed cadaver skull.

- Patient clinical trials. 


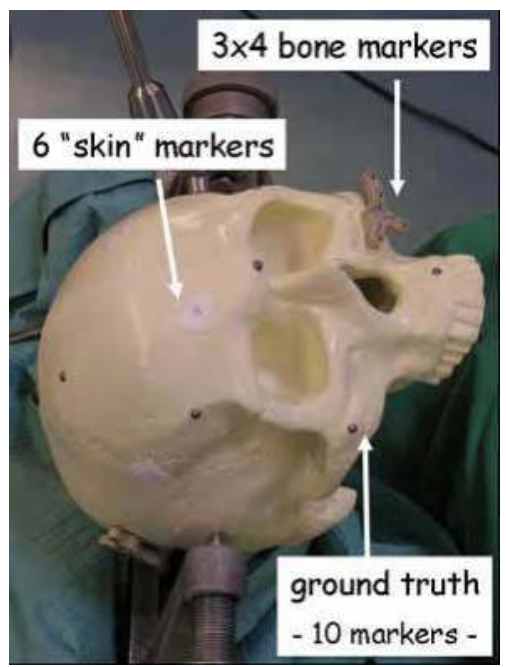

Figure 3. The laboratory set-up for phantom trials. Twelve bone markers ( $3 \times 4 \mathrm{BS})$ have been used for point based matching with implanted markers (Mode Ia). Further 10 markers have been used as the Ground Truth

The setup for the laboratory phantom trials is shown in Figure 3. The phantom was equipped with six adhesive skin markers, using the same placement procedure as in a standard neurosurgical patient preparation at Clinic for Neurosurgery, Ruhr-University Bochum. Three sets of four bone markers have been implanted for Mode Ia registration. Additional ten spherical bone markers (ø $4 \mathrm{~mm}$ ) served as the Ground Truth. The phantom skull was fixed in a standard Mayfield-clamp.

The cadaver skull was prepared with similar sets of four bone markers, implanted prior to the scanning (Figure 4). The same markers have been used for both, fiducial point based registration and ground truth, due to difficulties to implement further markers. Adhesive skin markers have been omitted for the reasons given in the further text. The cadaver skull was fixed in the Mayfield-clamp.

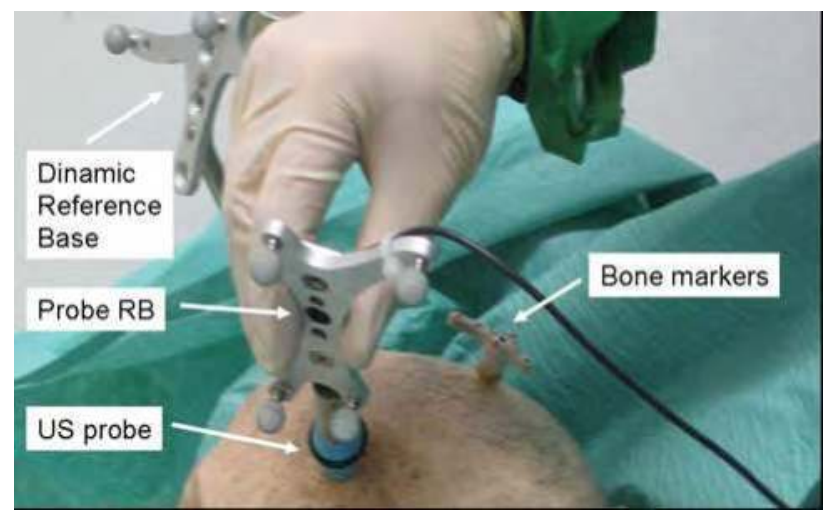

Figure 4. The laboratory set-up for cadaver trials 
For the clinical trials, a standard clinical procedure was utilized (Figure 5). Registration tests have been performed prior to intervention, after patient fixation and before sterilization, without interfering with the standard procedure.

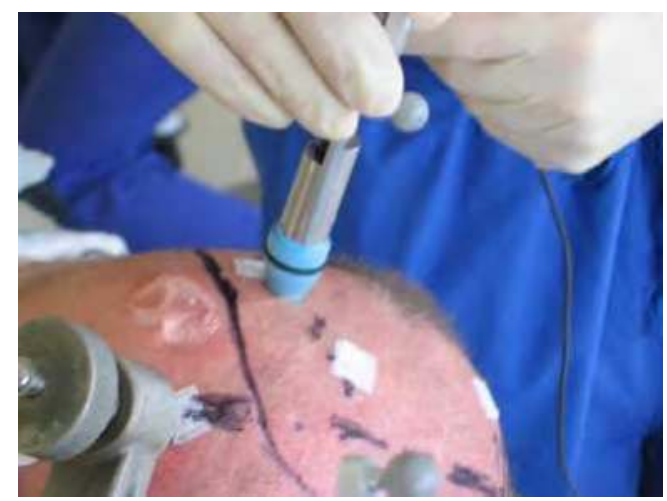

Figure 5. A clinical trial set-up

For all trials, the same radiological protocol has been used: slice thickness $=2 \mathrm{~mm}$, slice distance $2 \mathrm{~mm}$, pixel size $0.42 \mathrm{~mm}$.

\subsection{Ground Truth}

In order to evaluate the registration accuracy, titanium bone markers have been used. Due to a high contrast between the markers and the bone and high Hounsfield values of titanium, an automatic segmentation of titanium spheres is a trivial task. It was followed by an automatic sphere clustering and detection of centre of mass (i.e. geometrical centre) of each bone marker in the coordinate system of the CT scanner. Centre of each spherical marker is $\mathrm{g}_{\mathrm{i}} \mathrm{CT}$. After the registration, a transformation $\mathrm{T}_{\mathrm{R}}$, which maps points from the patient space to the coordinate system of the CT scanner, is obtained. The position of markers in patient's space coordinate system $\left(\mathrm{g}_{\mathrm{i}} \mathrm{P}\right)$ can be detection with a pointer, as described in 5.4. Therefore, the TRE in the i-th marker is:

$$
T R E_{i}=\left\|g_{i}^{C T}-T_{R}\left(g_{i}^{P}\right)\right\|
$$

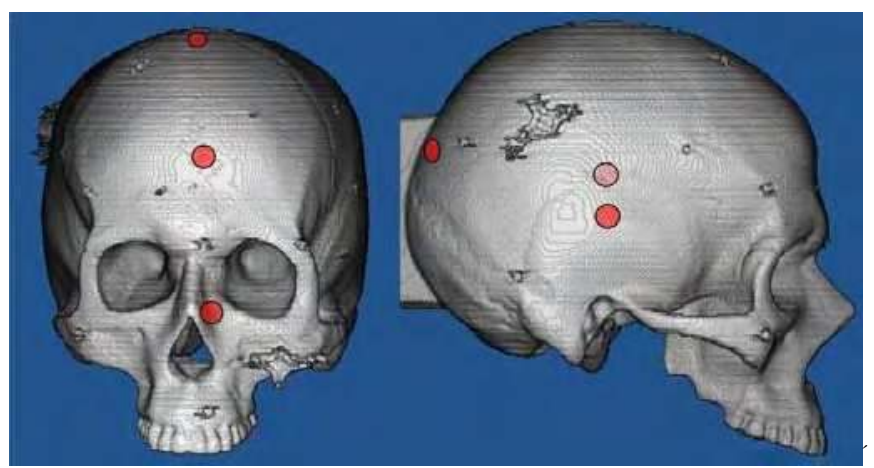

Figure 6. Points for the plausibility test (das Bild sieht aus, als wären da 6 Punkte) 


\subsection{Plausibility test}

Due to ethical reasons, to avoid discomfort to patients, no bone markers have been implanted for the clinical trials. In absence of fiducial bone markers, an alternative accuracy test (plausibility test) was performed. After a registration, bone surface points have been transcutaneously digitized at five uniformly distributed locations (Figure 6) using the US probe and subsequently have been transformed into the coordinate system of the CT dataset. The error was computed as the shortest distance to the closest point on the surface of the 3D model. This kind of error cannot account for different displacements of the transformation. However, the plausibility test might indicate a large registration error.

\subsection{Ultrasound system}

A-Mode ultrasound systems in general utilize single element transducers using a single channel pulser and receiver. The most common scanning method used for medical applications is the pulse-echo technique. Short pulses are sent into the tissue; a reflection profile, due to interfaces or small scatters, is received usually by the same transducer element. After amplification and digitization, the recorded A-Line can be further processed and analyzed. Interfaces are characterized by different acoustic impedance values, e.g. between bone and soft tissue. For a transcutaneous US-based registration, the objective is to detect a bone-soft tissue (skin) interface, in order to transcutaneously digitize the skull surface. The reflectivity of field intensity in case of the bone and soft tissue, assuming perpendicular sound incidence, is about $45 \%$. The distance between a transducer and a bone layer is calculated as $d=1 / 2 t \cdot v$, where $t$ is the delay of the US wave propagation through the tissue and $\mathrm{v}$ is sound velocity in soft tissue (1450-1630 m/s (Heger et al. 2005)).

In this study a $5 \mathrm{MHz}$, non-focused, heavily damped transducer with $5 \mathrm{~mm}$ element size is used. Based on the wavelength in water the measured focal distance for pulse excitation is approximately $17 \mathrm{~mm}$. The nominal $-6 \mathrm{~dB}$ beam width of the focus area is $1.2 \mathrm{~mm}$, which is equal to the theoretical lateral resolution.

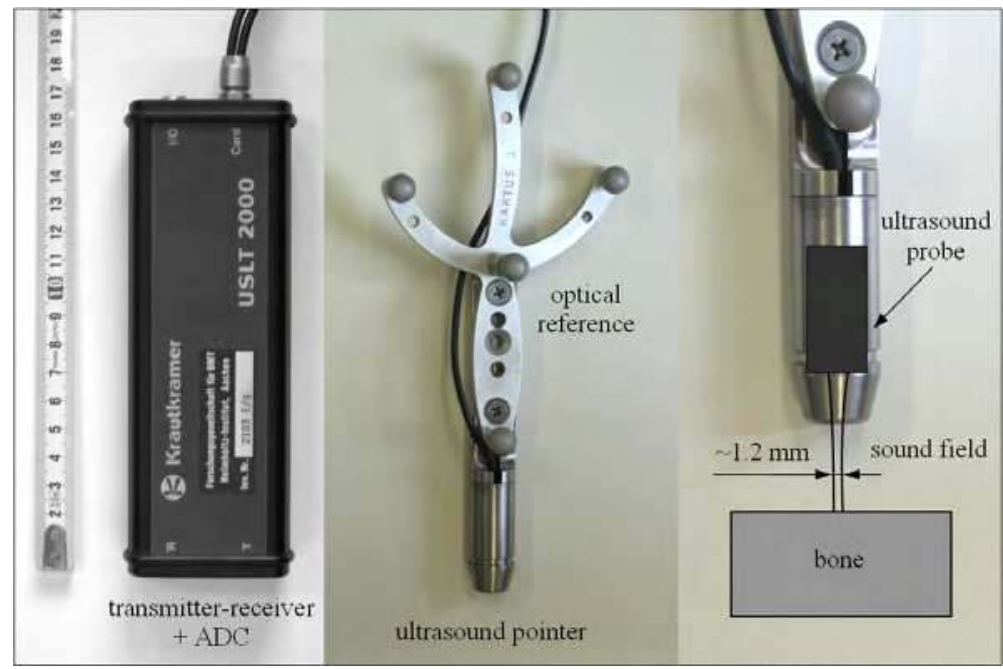

Figure 7. Portable single channel transmitter/receiver and ADC (left), A-mode Ultrasound pointer with optical reference (mid) and larger view of pointer and probe (right) 
A portable pulser/receiver hardware including analog to digital conversion (USLT 2000, Krautkramer, Germany) is used for the signal generation and basic signal processing (band pass filtering, signal amplification). A special autoclavable forerun is used to adapt the probe's working range to the typical thickness of skin tissue (Fig. 7). In order to receive a reliable ultrasound echo reflected at the bone tissue interface the beam axis of the transducer element has to be approximately perpendicular to the reflecting surface area. However, due to non-invasive palpation it is not possible for the user to see the local bone surface. Therefore, a perpendicular alignment of the ultrasound probe conventionally is difficult and time consuming, as reported by (Maurer 1999). For this reason a computer based guiding tool and a man-machine interaction algorithm has been developed to support the interactive alignment of the ultrasound transducer (Heger 2005). In order to simulate the intraoperative situation during the US registration on the phantom, i.e. the presence of the skin tissue, a piece of fresh meat is used to uncouple the probes' forerun and the phantom surface. In cadaver and patient studies, the forerun was applied directly to skin surface. The US probe was tracked using a 6-DOF optical tracking system.

\subsection{Tracking system}

A NDI Polaris System ${ }^{\circledR}$ for 6-DOF optical tracking of passive wireless rigid bodies has been used in a standard pyramid volume. Four different tools have been tracked: US probe, a pointed tool for AL, SM and surface-based registration, a Dynamic Reference Base (DRB) fixated at the Mayfield-clamp providing a dynamic coordinate system and allowing movement of the clamp during the registration, and a tool with a spherical tool-tip (ø $4 \mathrm{~mm})$ pivoted to the centre of the sphere, used for the Ground Truth. The rigid bodies have been equipped with at least four reflecting spheres. The rigid body at the US probe was optically pivoted to the surface of US transducer, along the central line of the probe. The probe with the rigid body is shown in Figure 7.

\section{Results}

\subsection{Accuracy}

For each mode in the phantom study, fifteen consecutive registrations were performed to assess the accuracy and robustness of the registration method. Each accuracy test was done by palpating ten accuracy markers. The mean accuracy is computed as follows:

$$
T R E_{m}=\frac{1}{N_{t}} \sum_{i=1}^{N_{t}}\left(\frac{1}{N_{m}} \sum_{j=1}^{N m} T R E_{i}^{j}\right)
$$

where $T R E_{i}^{j}$ is the TRE in the $\mathrm{i}$-th marker during the $\mathrm{j}$-th trial, $\mathrm{N}_{\mathrm{m}}$ is the number of markers (ten) and $\mathrm{N}_{\mathrm{t}}$ is the number of trials. Standard deviation of TRE is computed as:

$$
T R E_{S D}=\frac{1}{N_{t}} \sum_{i=1}^{N_{t}} \sqrt{\left(\frac{1}{N_{m}} \sum_{j=1}^{N m} T R E_{i}^{j}-T R E_{m}\right)^{2}}
$$

Table 2 shows the values achieved in the phantom study with ten repeated tests. 


\begin{tabular}{lllllll}
\hline & & Mode Ia & Mode Ib & Mode IIa & Mode IIb & Mode III \\
& & & & & & \\
\hline $\mathrm{TRE}_{\mathrm{m}}[\mathrm{mm}]$ & 0.58 & 1.78 & 2.78 & 1.38 & 1.37 \\
$\mathrm{TRE}_{\mathrm{SD}}[\mathrm{mm}]$ & 0.23 & 0.39 & 1.20 & 0.35 & 0.51 \\
$\mathrm{TRE}_{\max }[\mathrm{mm}]$ & 1.01 & 2.52 & 5.91 & 2.06 & 2.27 \\
$\mathrm{TRE}_{\min }[\mathrm{mm}]$ & 0.28 & 1.15 & 1.34 & 1.12 & 0.86 \\
\hline
\end{tabular}

Table 2. Accuracy values achieved in the phantom trial for different registration modes

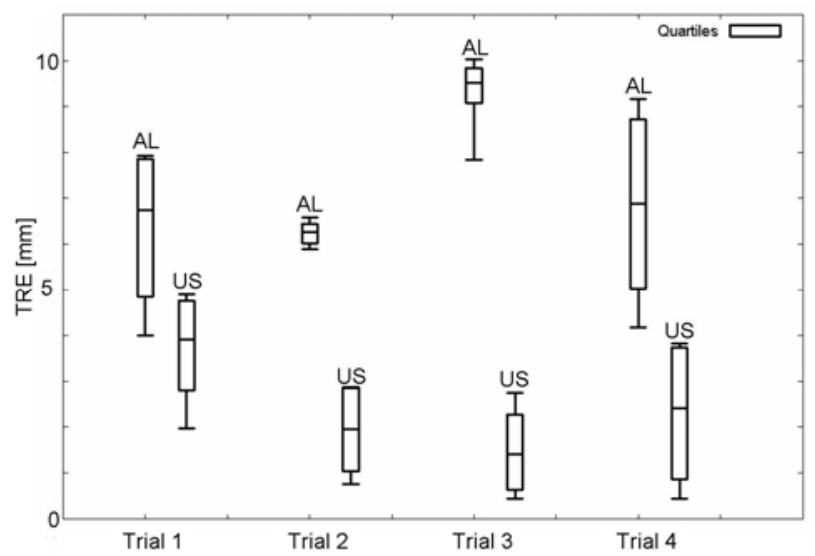

Figure 8. Box-Whisker-plot of four cadaver trials. A pre-registration with anatomical landmarks $(\mathrm{AL})$ is refined with an US surface-based registration

The accuracy using the adhesive skin markers in the phantom study (Table 2, column Ib) was significantly worse than surface-based registration (Table 2, columns IIb and III). Cadaver study is performed in modes Ia, IIa and III. A viable assumption is that accuracy of $\mathrm{SM}$ would decline if a cadaver is used, due to the presence of displaceable skin. Therefore, registration with $\mathrm{SM}$ in the cadaver study was omitted. Accuracy in mode Ia was similar as in the phantom study $\left(\mathrm{TRE}_{\mathrm{m}}=0.51, \mathrm{TRE}_{\mathrm{SD}}=0.18, \mathrm{TRE}_{\max }=0.97\right.$, and $\left.\mathrm{TRE}_{\min }=0.26\right)$. Results of AL and US-based registration for four cadaver trials are shown in Figure 8. It is important to notice that a pair of AL and US registration is subsequently performed (AL registration is used as a pre-registration for US surface-based matching). The US part of the plot is repeated in Figure 9 with a better resolution in y-axis.

\begin{tabular}{l|llllll}
\hline \multirow{2}{*}{ PATIENT } & Trial & P1 $[\mathrm{mm}]$ & P2 $[\mathrm{mm}]$ & P3 $[\mathrm{mm}]$ & P4 $[\mathrm{mm}]$ & P5 $[\mathrm{mm}]$ \\
\hline \multirow{2}{*}{ A } & 1 & 0.24 & 1.04 & 1.93 & 0.15 & 0.56 \\
& 2 & 2.88 & 1.91 & 1.75 & 1.34 & 0.87 \\
\hline B & 3 & 0.78 & 0.63 & 1.43 & 0.33 & 1.02 \\
C & 4 & 1.32 & 1.89 & 2.15 & 2.11 & 1.67 \\
\hline
\end{tabular}

Table 3. Plausibility test in five salient points (P1-P5) for three patients (A-C) 


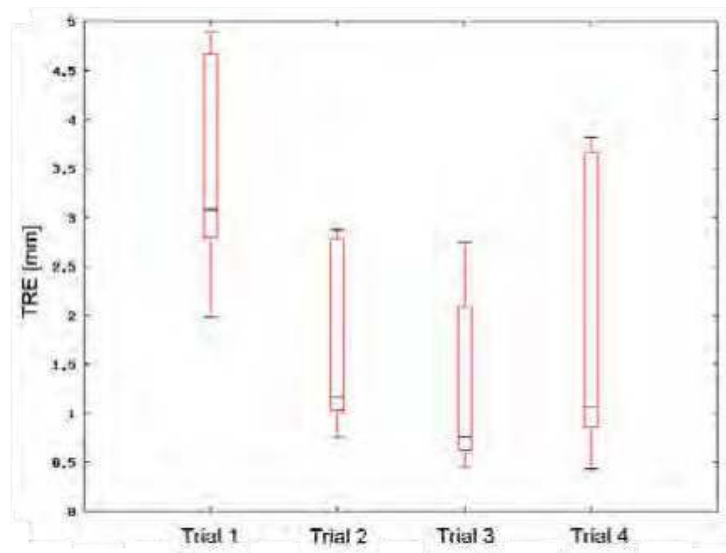

Figure 9. Box-Whisker-plot (quartiles) for four cadaver trials with A-mode US surface based registration

As mention before, in patient studies (three trials) the ground truth was not available, due to invasiveness of bone implantable markers. Therefore, a plausibility test, as described by the protocol in section 5.2 has been performed (Table 3). In order to analyse the results of the plausibility test, the Figure 10 shows a relation of accuracy and plausibility in the cadaver study.

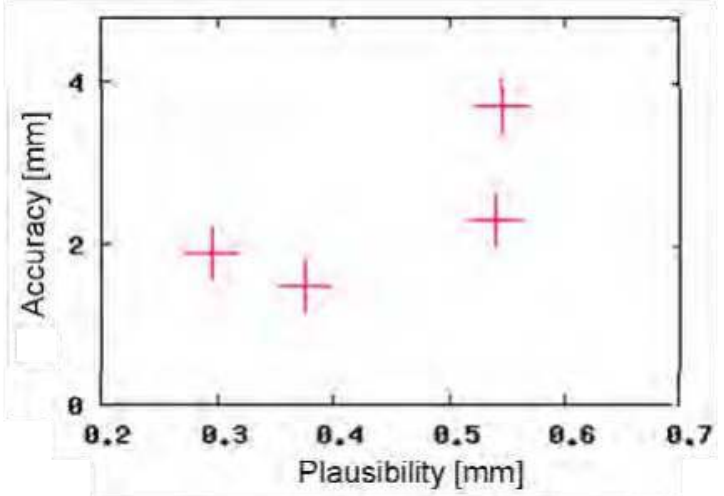

Figure 10. Relation between the plausibility and the accuracy in four cadaver trials

\subsection{Time efficiency}

The overall mean registration time using A-mode ultrasound (mean $=389 \mathrm{~s}$, SD 63s) is acceptable and smaller then the ones reported in the literature (15 min, Maurer 1999). The pre-registration with anatomical landmarks is less time critical (Figure 11) since it does not involve the US probe orientation. The ideal probe orientation is estimated from the 3D-CT model. During a registration, the user is supported with a graphical interface (Figure 12) showing an angular position of the probe to the surface normal vector. The majority of the time needed for US cadaver registration was spent for palpating the periorbital areas, due to significantly stiffer formalin-fixated soft tissue. 


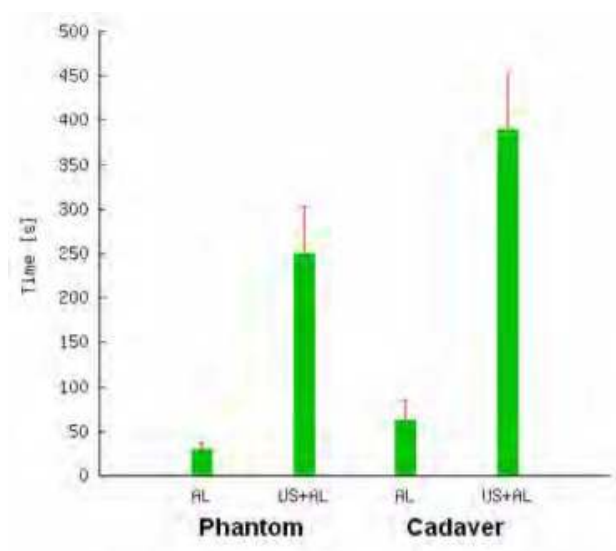

Figure 11. Time efficiency of the registration procedure

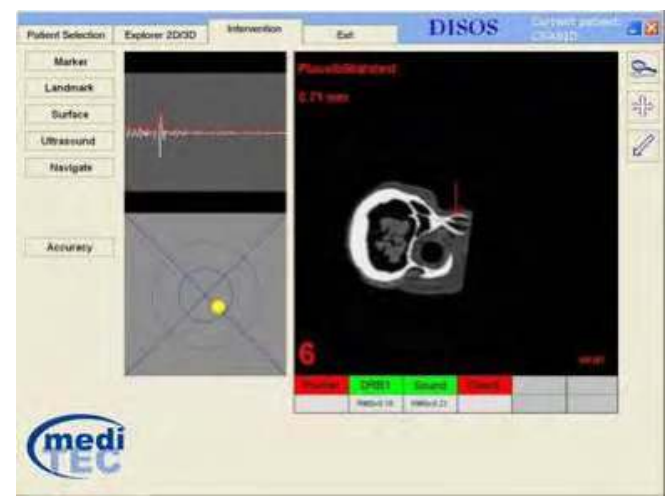

Figure 12. A screenshot of tool adjustment dialog

\section{Discussion}

The laboratory phantom tests have shown a significant correlation in error results between direct surface palpation and US-based registration indicating that the latter can be used in cases when direct palpation is not possible, e.g. in the presence of skin. The main limitation of surface based registration in neurosurgery is that only a small portion of bone is exposed during the operation. Therefore, the distribution of points can not follow a defined pattern, as described in section 2. The A-mode US transcutaneous registration offers two major improvements: palpation of a pervasive area of the skull surface and registration prior to sterilization (prior to skin opening). In comparison with anatomical landmarks, US-based surface registration has shown a significantly better accuracy $(1.37 \pm 0.51 \mathrm{~mm}$ vs. $2.78 \pm 1.20$ $\mathrm{mm}$ ). The result of $\mathrm{AL}$ registration was similar to those previously reported (e.g. (Wolfsberger et al. 2002)). Registration with adhesive skin markers has also shown poorer accuracy than US registration. As expected, registration with bone markers has shown an excellent accuracy in the range of CT pixel size $(0.58 \pm 0.23 \mathrm{~mm})$, but, as discussed previously, 
this technique requires an additional intervention prior to the surgery causing discomfort, possible skull infection and postoperative pain as well as additional costs.

Cadaver trials have shown a similar output as the phantom study. A significant improvement of the registration accuracy, if an US-based surface registration is used subsequently to AL registration, is shown (Figure 8). It is interesting to notice that the AL pre-registration accuracy is not significantly influencing the final result. For example, the worst AL registration accuracy has been observed in Trial 3. However, the final accuracy resulting from US surface-based matching, has shown the best performance in comparison with other trials. These results suggest that moderate pre-registration might be sufficient. However, a precaution in defining the palpation protocol should be taken, due to the local nature of the ICP algorithm.

Accuracy of the cadaver US-registration study has shown a promising mean and minimal values (1.97 and $0.63 \mathrm{~mm}$, respectively) with a strong standard deviation and poor maximal value (1.76 and $4.78 \mathrm{~mm}$, respectively). These results indicate that an acceptable registration with US-based technique is feasible; however, it should be done with a great care. A reason for inaccuracies in the cadaver study is an unusual ultrasound signal damping in the formalin-fixated soft tissue. Signal processing algorithms have been previously trained and tested on the normal tissue.

Due to lack of bone markers posing the ground truth, accuracy in patient studies could not have been assessed. The plausibility test was used as an indicator of registration goodness rather than an accuracy test. As Figure 10 indicates, there is a large discrepancy between the plausibility and accuracy. Therefore, although widely used, plausibility is of a questionable validity.

The overall mean registration time using A-mode ultrasound (mean $=389 \mathrm{~s}, \mathrm{SD} 63 \mathrm{~s}$ ) appear to be tolerable and smaller than the ones reported in the literature. The computer assisted alignment tool provides efficient support for US-based registration in cranial surgery. In vivo tests of US signal detection in regions where palpation was intended were more successful then in cadaver test, due to the stiffness of formalin-fixated soft tissue. Therefore, the majority of the time needed for US cadaver registration was spent for palpating the periorbital areas.

\section{Conclusions and Further Work}

In the first part of this chapter, theoretical background and practical considerations for the rigid registration of a patient dataset and a patient space in the operating theatre are given. Based on these, a surface palpation protocol has been developed and evaluated in three trials: phantom, cadaver and patient study.

The results have shown that A-mode US surface-based registration has a potential to be used in the clinical practice, due to its non-invasive nature and a better accuracy in comparison with other low invasive techniques (skin markers and anatomical landmarks). A user interface for computer guided probe orientation has shown time efficiency improvement, reducing total registration time to below seven minutes.

The major issue that needs to be address in the future is accuracy. Although registrations with acceptable accuracies have been demonstrated, the robustness remains questionable. It was shown that pre-registration plays a minor role in the overall accuracy. Therefore, the future work should focus on improving the palpation protocol and surface matching algorithms. Random ICP algorithm has shown promising results in both phantom and 
cadaver studies (Fieten et al. 2007). Furthermore, further development of the US signal processing is necessary to account for unusual tissue composition.

The registration techniques presented herein have been used in the CRANIO-System tests (Bast et al. 2006, Popovic et al. CARS 2007)

\section{References}

Arana, E., Martí-Bonmatí, L., Bautista, D., and Paredes, R (2004). Qualitative diagnosis of Calvarial metastasis by neural network and logistic regression. Academic Radiology, 11 (1):pp. 45-52, Jan 2004.

Bai, S., Teo, M., Ng, W., and Sim, C (2001). Workspace analysis of a parallel manipulator with one redundant DOF for skull-base surgery. In: Proceedings of the IEEE RSJ International Conference on Intelligent Robot and Systems, Outrigger Wailea Resort, Maui, Hawaii, USA. IEEE, 2001, pp. 1694-1699.

Bast, P., Engelhardt, M., Lauer, W., Schmieder, K., Rohde, V., and Radermacher, K. (2003) Identification of milling parameters for manual cutting of bicortical bone structures. Computer Aided Surgery, 8 (5):pp. 257-263, 2003.

Bast, P., Popovic, A., Wu, T., Heger, S., Engelhardt, M., Lauer, W., Radermacher, K., and Schmieder, K (2006). Robot- and computer-assisted craniotomy: resection planning, implant modelling and robot safety. The International Journal of Medical Robotics and Computer Assisted Surgery, 2 (2):pp. 168-178, June 2006.

Bernardete EA, Leonard MA, Weiner HL (2001). Comparison of frameless stereotactic systems: Accuracy, precision, and applications. Neurosurgery 2001;49:1409-1416.

Besl and McKay (1992): A method for registration of 3-D shapes. IEEE Trans. Pattern Anal. Mach. Intell., vol. 14, pp. 239-256, 1992.

Engelhardt, M., Bast, P., Jeblink, N., Lauer,W., Popovic, A., Eufinger, H., Scholz, M., Christmann, A., Harders, A., Radermacher, K., and Schmieder, K (2006). Analysis of surgical management of calvarial tumours and first results of a newly designed robotic trepanation system. Minimal Invasive Neurosurgery, 49 (2):pp. 98-103, April 2006.

Federspil, P. A., Geisthoff, U. W., Henrich, D., and Plinkert, P. K (2003). Development of the First force-controlled robot for otoneurosurgery. Laryngoscope, 113 (3):pp. 465-471, Mar 2003.

Federspil, P. A. and Plinkert, P. K (2004). Robotic surgery in otorhinolaryngology. Otolaryngol Pol, 58 (1):pp. 237-242, 2004

Fieten, L., Heger, S., Pasalic, L., Engelhardt, M., Schmieder, K., Radermacher, K. (2007) Registration of Cranial CT Images with A-Mode-Ultrasound Data Using the Random-ICP Algorithm -- A Cadaver Study, Proc. Biomedizinische Technik 2007

Fitzpatrick, M., West, J., Maurer, C. (1998) Predicting Error in Rigid-Body Point-Based Registration. IEEE Trans. on Medical Imaging, 17(5):694-702, 1998

Grunert, P., and J. Espinosa, K. D., and Filippi, R (2003). Computer-aided Navigation in Neurosurgery. Neurosurg Rev, 26:pp. 73-99, 2003.

Handini, D., Teo, M., and Lo, C (2004). System integration of NeuroBot: A skull-base Surgical robotic system. In: Proceedings of the IEEE Conference on Robotics, Automation and Mechatronics. December 2004, volume 1, pp. 43-48. 
Heger, S., F. Portheine, Jörg A. K. Ohnsorge, E. Schkommodau, K. Radermacher (2005). User Interactive Registration of Bone with A-Mode Ultrasound. IEEE Engineering in Medicine and Biology Magazine (M-EMB) March/April 2005; 85-95

Hill Derek and Philippe Batchelor (2005). Registration Methodology: Concepts and Algorithms. In Book: Medical Image Registration, CRC Press Inc., 2005

Laborde, G., Gilsbach, J., Harders, A., Klimek, L., Moesges, R., and Krybus, W (1992). Computer assisted localizer for planning of surgery and intra-operative orientation. Acta Neurochir (Wien), 119 (1-4):pp. 166-170, 1992.

Maurer, C., Jr. Ronal P. Gaston, D. L. G. Hill, M. J. Gleeson, M. G.Tayler, M. R. Fenlon, P. J. Edwards, D. J. Hawkes (1999). AcouStick: A Tracked A-Mode Ultrasonography System for Registration in Image-Guided Surgery. Proceedings MICCAI, Lecture Notes in Computer Science, 1679. (1999) 953-962

Nathoo, N., Cavusoglu, M. C., Vogelbaum, M. A., and Barnett, G. H (2005). In touch With robotics: neurosurgery for the future. Neurosurgery, 56 (3):pp. 421-33; discussion 421-33, Mar 2005.

Popovic, A., Engelhardt, M., Wu, T., Portheine, F., Schmieder, K., Radermacher, K (2003). CRANIO - Computer Assisted Planning for Navigation and Robot-assisted Surgery on the Skull. Computer Assisted Radiology and Surgery, Proceedings of the 17th International Congress and Exhibition, International Congress Series, 1256:1269-1276. Elsevier, June 2003.

Popovic, A., Wu, T., , Engelhardt, M., Radermacher, K (2006). In Medical Image Computing and Computer Assisted Intervention -- MICCAI 2006, Lecture Notes in Computer Science, 4191: 864-871. Springer, 2006.

Popovic, A., Wu, T., Follmann, A., Cunha Cruz, V.C.S., Schröder, K., Ibach, B., Engelhardt, M., Schmieder, K., Radermacher, K (2007). Cranio system for robot and computer assisted removal of calvarial tumors. In Computer Assisted Radiology and Surgery, Proceedings of the 19th International Congress and Exhibition (CARS 2007. International Journal of Computer Assisted Radiology and Surgery. Supp1 Vol 1 2007.

Radermacher K. (2006). Modeling of Intensity Priors for Knowledge-Based Level Set Algorithm in Calvarial Tumors Segmentation.

Schiffbauer H. (1999). Neuronavigation in brain tumor surgery Clinical beta-phase of the Oulu Neuronavigator System. Ph.D. thesis, Faculty of Medicine, University of Münster, 1999.

Spivak, C. J. and Pirouzmand, F. (2005) Comparison of the reliability of brain lesion localizationwhen using traditional and stereotactic image-guided techniques: a prospective study. J Neurosurg, 103 (3):pp. 424-427, Sep 2005.

Steinmeier R, Rachinger J, Kaus M, Gansalndt O, Huk W, Fahlbusch R (2002). Factors Influencing the application accuracy of neuronavigation systems. Stereotact Funct Neurosurg 2000;75: 188-202.

Tan TC, Black PM (2002). Sir Victor Horsley (1857-1916): pioneer of neurological surgery. Neurosurgery. 2002 Mar; 50(3):607-11

Tan, K. K., Grzeszczuk, R., Levin, D. N., Pelizzari, C. A., Chen, G. T., Erickson, R. K., Johnson, D., and Dohrmann, G. J (1993). A frameless stereotactic approach to neurosurgical planning based on retrospective patient-image registration. Technical note. J Neurosurg, 79 (2):pp. 296-303, Aug 1993. 
Watanabe (1996). Computer-integrated surgery: technology and clinical applications, MIT Press, Chapter: The neuronavigator: a computer-controlled navigation system in neurosurgery, pp. 319-327. 1996.

Watanabe, E., Watanabe, T., Manaka, S., Mayanagi, Y., and Takakura, K (1987). Three dimensional digitizer (neuronavigator): new equipment for computed tomographyguided stereotaxic surgery. Surg Neurol, 27 (6):pp. 543-547, Jun 1987.

Wolfsberger, S., K. Rössler, R. Regatschnig, K. Ungersböck (2002). Anatomical land-marks For image registration in frameless stereotactic neuronavigation. Neurosurg Rev 2002, Vol. 25, pp. 68-72

Wu T., Engelhardt M, Fieten L., Popovic A., Radermacher K.: Anatomically Constrained Deformation for Design of Cranial Implant: Methodology and Validation. In R. Larsen, M. Nielsen, and J. Sporring, editors, Medical Image Computing and Computer-Assisted Intervention (MICCAI 2006), vol. 4190 of Lecture Notes on Computer Science, pp. 9-16. Springer, 2006.

Zamorano, L., Kadi, A. M., and Dong, A (1992). Computer-assisted neurosurgery: simulation and automation. Stereotact Funct Neurosurg, 59 (1-4):pp. 115-122, 1992.

Zamorano, L. J., Nolte, L., Kadi, A. M., and Jiang, Z (1993). Interactive intraoperative Localization using an infrared-based system. Neurol Res, 15 (5):pp. 290-298, Oct 1993. 


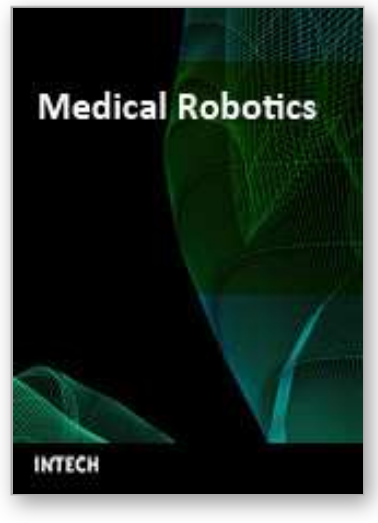

\author{
Medical Robotics \\ Edited by Vanja Bozovic
}

ISBN 978-3-902613-18-9

Hard cover, 526 pages

Publisher I-Tech Education and Publishing

Published online 01, January, 2008

Published in print edition January, 2008

The first generation of surgical robots are already being installed in a number of operating rooms around the world. Robotics is being introduced to medicine because it allows for unprecedented control and precision of surgical instruments in minimally invasive procedures. So far, robots have been used to position an endoscope, perform gallbladder surgery and correct gastroesophogeal reflux and heartburn. The ultimate goal of the robotic surgery field is to design a robot that can be used to perform closed-chest, beating-heart surgery. The use of robotics in surgery will expand over the next decades without any doubt. Minimally Invasive Surgery (MIS) is a revolutionary approach in surgery. In MIS, the operation is performed with instruments and viewing equipment inserted into the body through small incisions created by the surgeon, in contrast to open surgery with large incisions. This minimizes surgical trauma and damage to healthy tissue, resulting in shorter patient recovery time. The aim of this book is to provide an overview of the state-of-art, to present new ideas, original results and practical experiences in this expanding area. Nevertheless, many chapters in the book concern advanced research on this growing area. The book provides critical analysis of clinical trials, assessment of the benefits and risks of the application of these technologies. This book is certainly a small sample of the research activity on Medical Robotics going on around the globe as you read it, but it surely covers a good deal of what has been done in the field recently, and as such it works as a valuable source for researchers interested in the involved subjects, whether they are currently "medical roboticists" or not.

\title{
How to reference
}

In order to correctly reference this scholarly work, feel free to copy and paste the following:

Aleksandra Popovic, Stefan Heger, Axel Follmann, Ting Wu, Martin Engelhardt, Kirsten Schmieder and Klaus Radermacher (2008). Efficient Non-Invasive Registration with A-Mode Ultrasound in Skull Surgery, Medical Robotics, Vanja Bozovic (Ed.), ISBN: 978-3-902613-18-9, InTech, Available from: http://www.intechopen.com/books/medical_robotics/efficient_non-invasive_registration_with_amode_ultrasound_in_skull_surgery

\section{INTECH}

open science | open minds

\author{
InTech Europe \\ University Campus STeP Ri \\ Slavka Krautzeka 83/A
}

\author{
InTech China \\ Unit 405, Office Block, Hotel Equatorial Shanghai \\ No.65, Yan An Road (West), Shanghai, 200040, China
}


51000 Rijeka, Croatia

Phone: +385 (51) 770447

Fax: +385 (51) 686166

www.intechopen.com
中国上海市延安西路65号上海国际贵都大饭店办公楼 405 单元 Phone: +86-21-62489820

Fax: +86-21-62489821 
(C) 2008 The Author(s). Licensee IntechOpen. This chapter is distributed under the terms of the Creative Commons Attribution-NonCommercialShareAlike-3.0 License, which permits use, distribution and reproduction for non-commercial purposes, provided the original is properly cited and derivative works building on this content are distributed under the same license. 